\section{Comments on Tegaserod Trial on Irritable Bowel Syndrome}

TO THE EDITOR: Irritable bowel syndrome (IBS) remains to be a challenge for the gastroenterologist, due to its high prevalence and impacts on poor quality of life (QOL) with unsatisfactory pharmacological treatments. Most of the current treatment modalities for IBS have been directed at symptom relief rather than pathophysiology of the condition, which is heterogenous and poorly understood. ${ }^{1}$ I read the recent articles by Kim et al. ${ }^{2}$ about the tegaserod effects on IBS with great interest. The efficacy and safety of tegaserod have been demonstrated by several large randomized controlled trials. ${ }^{3,4}$ However, tegaserod was taken off the market by a high chance of having a myocardial infarction, stroke or angina. ${ }^{5}$

Kim et al. ${ }^{2}$ showed the efficacy of 5-hydroxytryptamine 4 $\left(5-\mathrm{HT}_{4}\right)$ agonist in Korean women with IBS with constipation. In this study, tegaserod showed the relief of overall IBS symptoms such as abdominal pain/discomfort, number of bowel movements and stool consistency. They used the composite score of symptom frequency and severity as an endpoint in treatment of IBS. The previously published pharmaceutical trial for IBS have used "adequate relief of abdominal pain and discomfort" or "satisfactory relief of IBS symptoms" as their primary outcome measure which led to approvals for alosetron and tegaserod by the Food and Drug Administration (FDA). ${ }^{6}$ An alternative method for defining a responder in an IBS treatment trial is to ask patients to report the frequency and severity of all IBS symptoms. Kim et al. ${ }^{2}$ showed the adequate symptom relief and good correlation between symptom composite score and IBS-QOL, which might show the usefulness of Korean IBS-QOL in IBS therapeutic trial. However, it is not clear whether reduction of sum-score of 22.5/96 (23.5\%) was enough to define a responder. They conducted this trial as open arm without placebo control. The FDA have recommended investigators to provide rules, a priori, which allow classification of each participant as a responder or non-responder for the primary outcome. ${ }^{7}$ The secondary outcome is used to strengthen the results by showing concordance between individual symptoms and the primary outcome measure, addressing the mechanism of the intervention, and assessing the safety. ${ }^{6}$ Kim et al. ${ }^{2}$ also proposed QOL to be included as a therapeutic outcome.

Recently, there are many pharmaceutical trials including the next generation 5-HT agonists, such as Prucalopride, TD-5108, and ATI-7505 in IBS. ${ }^{8}$ I am hoping for the present study to strengthen the pharmaceutical research in IBS. The primary outcome variables provide the basis for judging the success or failure of an intervention, therefore, further studies on the outcome measurements in IBS drug trials, which can properly quantify drug responses, are warranted.

Hye-Kyung Jung, MD

Department of Internal Medicine Ewha Medical Research Institute Ewha Womans University School of Medicine Seoul, Korea

1. Chey WY, Jin HO, Lee MH, Sun SW, Lee KY. Colonic motility abnormality in patients with irritable bowel syndrome exhibiting abdominal pain and diarrhea. Am J Gastroenterol 2001;96:1499-1506.

2. Kim YS, Choi SC, Park JM, et al. The effect of tegaserod on symptoms and quality of life in Korean women with irritable bowel syndrome with constipation. J Neurogastroenterol Motil 2010;16:61-70.

3. Müler-Lissner SA, Fumagalli I, Bardhan KD, et al. Tegaserod, a 5-HT(4) receptor partial agonist, relieves symptoms in irritable bowel syndrome patients with abdominal pain, bloating and constipation. Aliment Pharmacol Ther 2001;15:1655-1666.

4. Novick J, Miner P, Krause R, et al. A randomized, double-blind, placebo-controlled trial of tegaserod in female patients suffering from irritable bowel syndrome with constipation. Aliment Pharmacol Ther. 2002;16:1877-1888.

5. Fayyaz M, Lackner JM. Serotonin receptor modulators in the treatment of irritable bowel syndrome. Ther Clin Risk Manag 2008;4: 41-48.

6. Design of Treatment Trials Committee, Irvine EJ, Whitehead WE, et al. Design of treatment trials for functional gastrointestinal disorders. Gastroenterology 2006;130:1538-1551.

7. Committee for Proprietary Medicinal Products (CPMP) Committee for Proprietary Medicinal Products (CPMP): points to consider on adjustment for baseline covariates. Stat Med 2004;23: 701-709.

8. Shekhar C, Whorwell PJ. Emerging drugs for irritable bowel syndrome. Expert Opin Emerg Drugs 2009;14:673-685.

\section{Conflicts of interest: None.}

DOI: 10.5056/jnm.2010.16.2.222

Reply. We appreciate the comments from Dr. Hye-Kyung Jung. There have been several issues in clinical trials on irritable bowel syndrome (IBS) such as duration of trial, study design, on demand or repeated cycles of treatment, and optimal efficacy endpoint. ${ }^{1,2}$ Among these, optimal endpoint issue is one of the most important factors to prove efficacy of therapeutic agent in a clinical trial, ${ }^{3}$ however, widely accepted and validated outcome 
measures is unavailable at present. Contrary to other gastrointestinal diseases such as gastroesophageal reflux disease or inflammatory bowel disease which are diagnosed and assessed by endoscopy, laboratory, and radiologic evaluation, successful treatment of IBS in outpatient office or assessment of effect in IBS clinical trial could be measured only by symptom improvement. ${ }^{4}$

Binary endpoints, such as adequate relief and satisfactory relief, have been used most commonly as primary endpoint in most IBS clinical trials. However there is a question whether it is enough as primary endpoint ${ }^{5,6}$ therefore it should be reinforced by secondary (supportive) efficacy endpoints in the clinical research. Although IBS symptom and quality of Life (QOL) score could be used as secondary endpoint, the utility of QOL score in IBS clinical trial has not been explored as satisfactory ${ }^{7}$ and some authors suggest that QOL score should be considered as a tertiary endpoint. ${ }^{4}$

One of the important purposes of our study was to validate the usefulness of QOL as an endpoint and we found that QOL score was comparable to symptom score in IBS clinical trial. QOL provides important information to clinicians about the aspects of health care that "actually get to the patient" European Agency for the Evaluation of Medicinal Product and Rome III committee emphasized QOL assessments as important secondary outcomes. ${ }^{2,9}$

The problem is that there has been no consensus about the definition of responder in the outcomes by symptoms or QOL scoring system. We were also troubled with the definition of "clinically meaningful change" in scoring system when we designed our study protocol. The magnitude of score changes which means clinically important improvement in individual symptom or QOL has not been defined in IBS trials. ${ }^{2}$ Some studies considered as little as $10 \%$ reduction in visual analog scale rating of symptom severity ${ }^{10}$ or 1 step on 7 -step ordinal scale ${ }^{9}$ as clinically meaningful, whereas other studies used $50 \%$ reduction in an aggregate symptom severity index ${ }^{11}$ or questionnaire. ${ }^{5} \mathrm{~A}$ statistically significant $\mathrm{p}$-value also does not imply whether a particular finding is "clinically meaningful change."

Therefore we recommend clinical researchers to use QOL as an endpoint in IBS clinical trials and hope that further studies will be carried out to figure out what is the magnitude of "clinically meaningful change" in symptoms and QOL scoring system.

Yong Sung Kim, MD

Suck Chei Choi, MD

Department of Internal Medicine Wonkwang Digestive Disease Research Institute Wonkwang University School of Medicine Iksan, Jeollabuk-do, Korea

1. Corazziari E, Bytzer P, Delvaux M, et al. Clinical trial guidelines for pharmacological treatment of irritable bowel syndrome. Aliment Pharmacol Ther 2003;18:569-580.

2. Design of Treatment Trials Committee, Irvine EJ, Whitehead WE, et al. Design of treatment trials for functional gastrointestinal disorders. Gastroenterology 2006;130:1538-1551.

3. Camilleri M, Mangel AW, Fehnel SE, Drossman DA, Mayer EA, Talley NJ. Primary endpoints for irritable bowel syndrome trials: a review of performance of endpoints. Clin Gastroenterol Hepatol 2007;5:534-540.

4. Schoenfeld P, Talley NJ. Measuring successful treatment of irritable bowel syndrome: is "satisfactory relief" enough? Am J Gastroenterol 2006;101:1066-1068.

5. Whitehead WE, Palsson OS, Levy RL, Feld AD, VonKorff M, Turner M. Reports of "satisfactory relief” by IBS patients receiving usual medical care are confounded by baseline symptom severity and do not accurately reflect symptom improvement. Am J Gastroenterol 2006;101:1057-1065.

6. Passos MC, Lembo AJ, Conboy LA, et al. Adequate relief in a treatment trial with IBS patients: a prospective assessment. Am J Gastroenterol 2009;104:912-919.

7. Lembo A, Ameen VZ, Drossman DA. Irritable bowel syndrome: toward an understanding of severity. Clin Gastroenterol Hepatol 2005;3:717-725.

8. Koller M, Neugebauer EA, Augustin M, et al. Assessment of quality of life in health services research - conceptual, methodological and structural prerequisites. Gesundheitswesen 2009;71:864-872.

9. Committee for Proprietary Medicinal Products (CPMP). Committee for Propriety Medicinal Products (CPMP): points to consider on adjustment for baseline convariates. Stat Med 2004;23:701-709.

10. Bardhan KD, Bodemar G, Geldof $\mathrm{H}$, et al. A double-blind, randomized, placebo-controlled dose-ranging study to evaluate the efficacy of alosetron in the treatment of irritable bowel syndrome. Aliment Pharmacol Ther 2000;14:23-34.

11. Payne A, Blanchard EB. A controlled comparison of cognitive therapy and self-help support groups in the treatment of irritable bowel syndrome. J Consult Clin Psychol 1995;63:779-786.

\section{Conflicts of interest: None.}

DOI: 10.5056/jnm.2010.16.2.223 